or nitrite can increase plasma cGMP and reduce blood pressure $(15,17,18)$. However, like any potential therapy, there are potential adverse effects $(15,18)$. Dietary supplementation of nitrites can cause methemoglobinemia and interfere with oxygenation of the blood. Furthermore, dietary nitrite and nitrate can contribute to the formation of potentially carcinogenic nitrosamines, although the epidemiological association of dietary nitrogen oxides with cancer has been questioned (19). Accordingly, until there is more guidance from larger, randomized clinical trials, direct supplementation of the diet with nitrite or nitrate salts seems inadvisable. Adherents of the hypothesis may be served best by generous helpings of leafy green vegetables.

\section{Acknowledgments}

The authors thank Jonathan Stamler for comments on this work. This work was supported in part by grants from the NIH (RC2HL103400, 1U01HL100397, and K12 HL087746) and by the Tobacco-Related Disease Research Program of the University of California (18XT-0098).
Address correspondence to: John P. Cooke, Stanford Cardiovascular Institute, 300 Pasteur Drive, A260 MC 5319, Stanford, California 94305, USA. Phone: 650.723.6459; Fax: 650.723.8392; E-mail: john.cooke@ stanford.edu.

1. Kots AY, Martin E, Sharina IG, Murad F. A short history of cGMP, guanylyl cyclases, and cGMPdependent protein kinases. Handb Exp Pharmacol. 2009;191(191):1-14.

2. Lima B, Forrester MT, Hess DT, Stamler JS. S-nitrosylation in cardiovascular signaling. Circ Res. 2010;106(4):633-646.

3. Cooke JP. Flow, NO, and atherogenesis. Proc Natl Acad Sci U S A. 2003;100(3):768-770.

4. Cooke JP. Asymmetrical dimethylarginine: the Uber marker? Circulation. 2004;109(15):1813-1818.

5. Münzel T, Gori T, Bruno RM, Taddei S. Is oxidative stress a therapeutic target in cardiovascular disease? Eur Heart J. 2010;31(22):2741-2748.

6. Patel SD, Waltham M, Wadoodi A, Burnand KG, Smith A. The role of endothelial cells and their progenitors in intimal hyperplasia. Ther $A d v$ Cardiovasc Dis. 2010;4(2):129-141.

7. McDonald OG, Owens GK. Programming smooth muscle plasticity with chromatin dynamics. Circ Res. 2007;100(10):1428-1441.

8. Morris SM Jr. Recent advances in arginine metabolism: roles and regulation of the arginases. $\mathrm{BrJ}$ Pharmacol. 2009;157(6):922-930.

9. Xia Y, Dawson VL, Dawson TM, Snyder SH, Zweier JL. Nitric oxide synthase generates superoxide and nitric oxide in arginine-depleted cells leading to peroxynitrite-mediated cellular injury. Proc Natl Acad Sci U S A. 1996;93(13):6770-6774.

10. Lassègue B, Griendling KK. NADPH oxidases: functions and pathologies in the vasculature. Arterioscler Thromb Vasc Biol. 2010;30(4):653-661.

11. Alef MJ, et al. Nitrite-generated NO circumvents dysregulated arginine/NOS signaling to protect against intimal hyperplasia in Sprague-Dawley rats. J Clin Invest. 2011;121(4):1646-1656.

12. Feelisch $M$, et al. Tissue processing of nitrite in hypoxia: an intricate interplay of nitric oxide-generating and -scavenging systems. J Biol Chem. 2008; 283(49):33927-33934.

13. Zweier JL, Li H, Samouilov A, Liu X. Mechanisms of nitrite reduction to nitric oxide in the heart and vessel wall. Nitric Oxide. 2010;22(2):83-90.

14. Lundberg JO, et al. Nitrate and nitrite in biology, nutrition and therapeutics. Nat Chem Biol. 2009; 5(12):865-869.

15. Weitzberg E, Hezel M, Lunderg JO. Nitratenitrite-nitric oxide pathway: Implications for anesthesiology and intensive care. Anesthesiology. 2010;113(6):1460-1475.

16. Dykhuizen RS, et al. A clinical trial of the effects of dietary patterns on blood pressure. DASH Collaborative Research Group. N Engl J Med. 1997; 336(16):1117-1124

17. Kapil V, et al. Inorganic nitrate supplementation lowers blood pressure in humans: role for nitritederived NO. Hypertension. 2010;56(2):274-281.

18. Tang Y, Jiang H, Bryan NS. Nitrite and nitrate: cardiovascular risk - benefit and metabolic effect. Curr Opin Lipidol. 2011;22(1):11-15.

19. Powlson DS, et al. When does nitrate become a risk for humans? J Environ Qual. 2008;37(2):291-295.

\title{
Neutrophils give us a shock
}

\section{Clifford A. Lowell}

\author{
Department of Laboratory Medicine, UCSF, San Francisco, California, USA.
}

\begin{abstract}
Systemic anaphylaxis is generally recognized as a severe allergic reaction caused by IgE-mediated activation of mast cells, leading to massive release of vasoactive mediators that induce acute hypotension and shock. However, experimental evidence in mice suggests that this view is too simple. Using a variety of techniques to manipulate immune cell makeup, Jönsson et al. come to the conclusion in this issue of the JCI that recognition of IgG1 and IgG2 antibodies by $F c \gamma$ RIII and Fc $\gamma$ RIV receptors on neutrophils is a major pathway for induction of anaphylaxis. These exciting results suggest that we have to reevaluate our models for anaphylaxis in humans, which will have a direct impact on our therapeutic approaches for prevention of this potential deadly hypersensitivity reaction.
\end{abstract}

I turned to Wikipedia when I was searching for a way to explain the basics of anaphylaxis and came across the following statement: "True anaphylaxis is caused by degranulation of mast cells or basophils mediated by immunoglobulin E (IgE)." This is the classic teaching, present in all

Conflict of interest: The author has declared that no conflict of interest exists.

Citation for this article: J Clin Invest. doi:10.1172/ JCI57296. the immunology textbooks, but the paper in this issue of the JCI by Jönsson et al. (1) informs us that this view is, at best, incomplete. Instead, we learn that anaphylaxis can be mediated by neutrophils recognizing IgG/antigen complexes. In addition to turning around our understanding of anaphylaxis, this paper adds to the growing list of neutrophil functions besides just bacterial killing and protease production (2). Lately we have learned that neutrophils are major sourc- es of cytokines and chemokines $(3,4)$. They play a direct role in influencing the recruitment and activation of monocytes/ macrophages, T cells, and NK cells during inflammation (5-7). Neutrophils have been implicated as the primary initiators of immune complex-mediated diseases $(8,9)$. And now the shocking news (pun intended!) that they are major players in initiating anaphylaxis.

\section{The history of anaphylaxis}

Anaphylaxis is an acute, multisystem, severe type I hypersensitivity reaction that develops in minutes to hours following antigen exposure (10). In its mildest forms, it results in rashes (hives), wheezing, and some gastrointestinal symptoms (cramping, bloating). In its more severe forms, patients develop bronchoconstriction with hypoventilation, systemic vasodilation (leading to frank shock), cardiac dysrhythmias, and central nervous system abnormalities. Anaphylaxis most often 


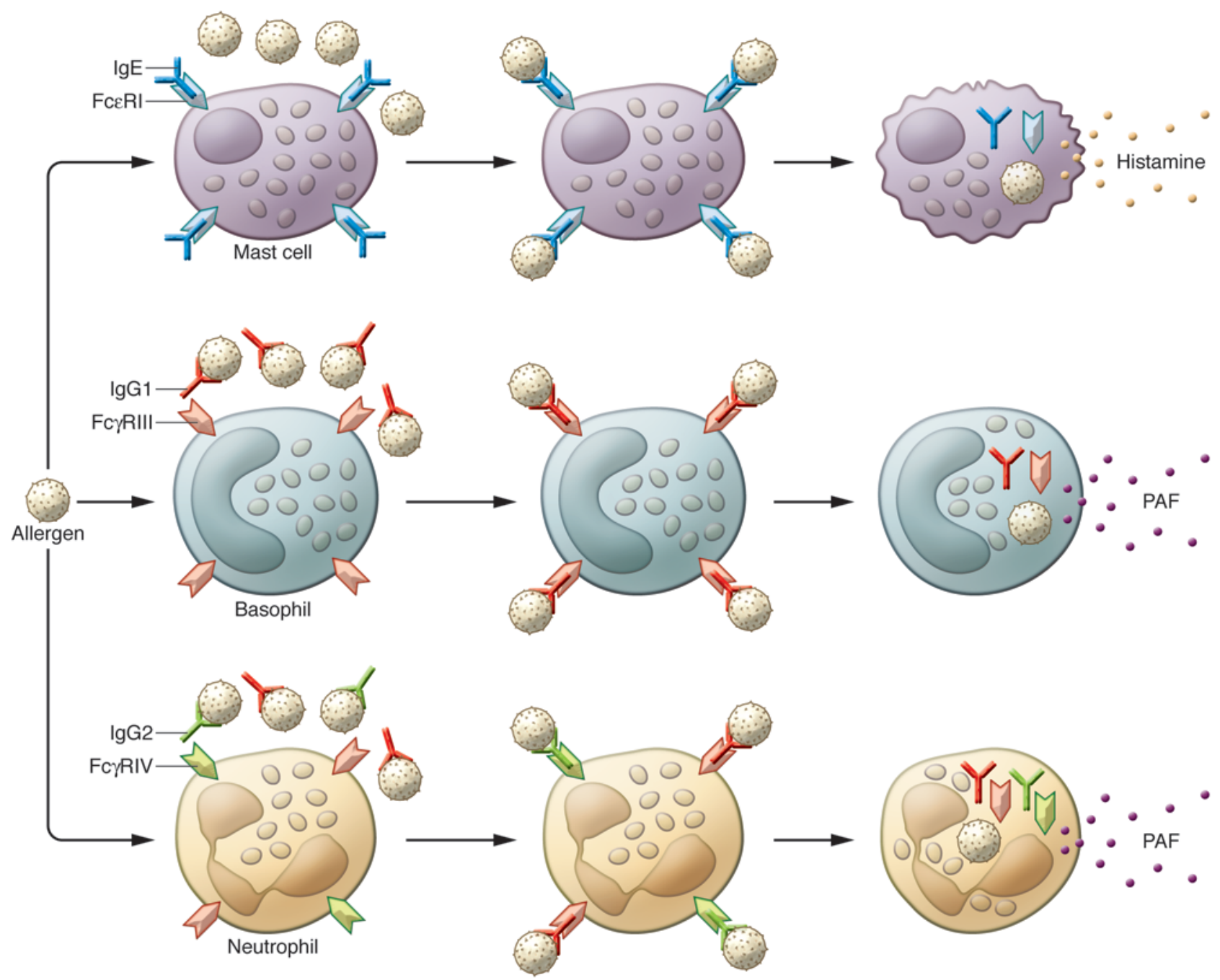

Figure 1

Mast cells, basophils, and neutrophils in anaphylaxis. Following allergen exposure, IgE and IgG Abs are produced. IgE binds to FcERs on mast cells, priming the cells for a secondary response. Following second exposure, the allergen binds to IgE on mast cell receptors, activating histamine release. Allergens also form immune complexes with IgG1- and IgG2-activating basophils and neutrophils through FcyRIII and Fc $\gamma \mathrm{RIV}$, respectively, leading to PAF release. Neutrophils also express F $\gamma \gamma R$ III, and they also respond to IgG1/allergen complexes.

occurs in patients with severe allergy to insect stings (specifically Hymenoptera venom), specific foods (mainly nuts, shellfish, some milk products), or in response to some medications. Amazingly, it is estimated that $1 \%-15 \%$ of people in the US are at risk for anaphylaxis-type reactions and that upwards of 1,500 deaths per year are attributed to acute hypersensitivity reactions $(11,12)$.

Experimentally, anaphylaxis is studied in two fashions. Active systemic anaphylaxis (ASA) is induced by immunizing experimental animals, then rechallenging them with the antigen in a form that induces an acute hypersensitivity response; this model closely mimics human anaphylaxis. Passive systemic anaphylaxis (PSA) involves adoptive transfer of antigen-specific Abs into naive animals followed by injection of the antigen. In both cases, use of knockout mice lacking various immune cells, receptors, or signaling molecules has allowed investigators to dissect the mechanisms of hypersensitivity reactions, as exemplified by Jönsson et al. (1).

\section{A Nobel Prize for anaphylaxis - making lemonade out of lemons}

The term anaphylaxis was coined by Charles Richet, a French physiologist, in his work with colleague Paul Portíer, trying to establish immunity in dogs to sea anemone toxin (13). Their objective was to make the animals tolerant to the toxin by first injecting them with nonlethal doses, followed by subsequent challenge doses. This protective effect of prior exposure had been demonstrated with other toxins in other animal species. To their dismay, Portíer and Richet found that their dogs developed lethal hypersensitivity reactions within minutes following a second injection of even small doses of the toxin. Portíer and Richet coined a new term, anaphylaxis, derived from the Greek words a (against) and phylaxis (protection), to describe what appears today to be a failed experiment. This work was published in 1902 (14), and Richet received the Nobel Prize in Medicine/ Physiology in 1912. That is making lemonade out of lemons, indeed! 


\section{The mast cell as the initiator of anaphylaxis?}

A century after Portíer and Richet's work, our molecular understanding of anaphylaxis is that it is an $\mathrm{IgE} /$ mast cell/basophil-mediated event (reviewed in ref. 10). Sensitized individuals develop antigenspecific IgE, which binds to FceR receptors on mast cells and basophils, priming the cells for robust responses. Following reexposure to the allergen, FceRs are aggregated on the responding cells, leading to degranulation and release of "preformed" mediators, principally histamine, which in turn induces the symptoms of allergy, or in its most systemic form, anaphylaxis (Figure 1). There is a wealth of experimental data supporting this model, including years of experiments in defining the "reagin" of allergy as the immunoglobulin IgE, then the cloning of the Fce R receptors and demonstrating their presence on mast cells, along with the recognition of mast cell products as having direct vascular effects that can lead to hypotension and shock, which characterizes severe anaphylaxis. It is also clear that many other mast cell products may contribute to allergy and anaphylaxis, including proteoglycans, serine proteases, lipid-derived mediators, cytokines, and platelet-activating factor (PAF) (10).

With more modern approaches in mouse models, it becomes clear that this simple paradigm is unlikely to be the entire story. Using the PSA model in mice, it is well established that administration of either IgE or IgG1 can cause reactions following antigen challenge, with IgE acting through FceR and IgG1 acting through FcyRIII receptors (15). Using basophildepleting $\mathrm{mAbs}$ and mast cell-deficient mice, it appears that the IgG1 responses are initiated primarily by Fc $\gamma$ RIII receptors on basophils, which release large amounts of PAF (16). More importantly, it has long been recognized that ASA can be induced in mice lacking either mast cells or IgE, suggesting that other pathways must be operative $(17,18)$. ASA can also be induced in basophil-depleted animals (16), but fails to occur in mice lacking activating Fc $\gamma$ Rs (15). Moreover, blockade of the PAF receptor limits peanut-induced allergy in mouse models (19). These findings lead us to conclude that antibodies other than IgE can mediate ASA, that cells other than mast cells or basophils are involved, and that histamine is not the only mediator of anaphylaxis.

\section{The $5 \mathrm{KO}$ mouse and new blocking Abs}

Jönsson et al. (1) address these issues using a panel of relatively new knockout mouse models as well as some novel blocking mAbs. Their basic model involves immunization of mice with BSA in Freund's adjuvant or alum, followed by confirmation of IgE and IgG anti-BSA titers, then subsequent rechallenge with intravenously injected BSA. The animals develop a fatal ASA within minutes and can be monitored by a drop in core body temperature. Using this simple model, the authors demonstrate that ASA still develops in $5 \mathrm{KO}$ mice, which are derived by interbreeding of 5 different knockout strains and lack all IgE FceRs and all IgG Fcy Rs except FcyRIV, which is present only on neutrophils and macrophages. Treatment of mice with anti-Fc $\gamma$ RIV blocks the ASA in $5 \mathrm{KO}$ mice, and in combination with a new antiFc $\gamma$ RIII-blocking mAbs, ASA is blocked in regular wild-type mice. Macrophage depletion does not protect mice in their ASA model, but neutrophil depletion (especially combined with loss of basophils) is fully protective. The type of adjuvant used in the immunization matters, with alum favoring production of IgG1 antibodies, which then results in loss of ASA in the $5 \mathrm{KO}$ strain. The authors conclude that BSA-specific IgG1 binding to Fc $\gamma$ RIII on basophils (and likely other cells) and IgG2 binding to Fcy RIV on neutrophils is sufficient to mediate ASA. Amazingly, the authors confirm the neutrophil dominance in their ASA model by demonstrating that adoptive transfer of human neutrophils into mice that lack all activating FceRs and $\mathrm{Fc} \gamma \mathrm{Rs}$ restores normal reactions to BSA challenge. They confirm the relative specificity of IgG1 to FcyRIII versus IgG2 to Fc $\gamma$ RIV in PSA-type experiments, using different anti-DNP mAbs. They also confirm the neutrophil dominance in PSA using a polyclonal $\mathrm{Ab}$ model, performed by injecting mice with preformed immune complexes. Finally, using inhibitors and cPLA2 mutant mice that fail to make PAF, they demonstrate that PAF, but not histamine, seems to be the dominant mediator in their ASA model.

\section{Caveats and future directions}

Although this is an outstanding and wellcontrolled study, there are always caveats. There are minor issues, such as the need to test ASA and IgG2 PSA in newly developed FcyRIV KO mice (20), which will be quickly done. The studies involving PAF as a major mediator are somewhat limited at this point. There is also the general concern that all these experiments are done with substantial immunization protocols (such as Freund's adjuvant) followed up by injection of large amounts of antigen, paradigms that do not recapitulate the development of lethal hypersensitivity in a child following a single bee sting. Moreover, the repertoire and expression pattern of Fc $\gamma$ Rs differs significantly between mouse and humans, so it remains generally unknown how translatable mouse models for anaphylaxis are to humans. Nevertheless, it is clear that physicians and scientists need to pay more attention to anti-specific IgGs and neutrophils in patients with hypersensitivity reactions. Therapeutically, this could translate into more effort put toward blocking IgG or $\mathrm{Fc} \gamma \mathrm{R}$ binding or inhibiting PAF function in patients with severe allergy. Finally, one thing is for sure: this wonderful study by Jönsson et al. (1) will mean that somebody has to sit down and update Wikipedia; hopefully, by the time you have finished reading this paper, it will be done!

\section{Acknowledgments}

The author's work is supported by the NIH grants AI65495 and AI68150.

Address correspondence to: Clifford A. Lowell, Department of Laboratory Medicine, University of California, San Francisco, 513 Parnassus Ave., MSB-1058, California, 94143-0451, USA. Phone: 415.476.2963; Fax: 415.502.5462; E-mail: clifford.lowell@ucsf.edu.

1. Jönsson F, et al. Mouse and human neutrophils induce anaphylaxis. J Clin Invest. 2011; 121(4):1484-1496.

2. Borregaard N. Neutrophils, from marrow to microbes. Immunity. 2010;33(5):657-670.

3. Scapini P, Lapinet-Vera JA, Gasperini S, Calzetti F, Bazzoni F, Cassatella MA. The neutrophil as a cellular source of chemokines. Immunol Rev. 2000; 177:195-203.

4. Zhang X, Majlessi L, Deriaud E, Leclerc C, Lo-Man R. Coactivation of Syk kinase and MyD88 adaptor protein pathways by bacteria promotes regulatory properties of neutrophils. Immunity. 2009;31(5):761-771.

5. Soehnlein O, Lindbom L. Phagocyte partnership during the onset and resolution of inflammation. Nat Rev Immunol. 2010;10(6):427-439.

6. Costantini C, Cassatella MA. The defensive alliance between neutrophils and NK cells as a novel arm of innate immunity. J Leukoc Biol. 2011;89(2):221-233.

7. Pelletier M, et al. Evidence for a cross-talk between human neutrophils and Th17 cells. Blood. 2010; 115(2):335-343

8. Mancardi DA, et al. Cutting Edge: The murine high-affinity IgG receptor FcyRIV is sufficient for autoantibody-induced arthritis. J Immunol. 2011; 
186(4):1899-1903.

9. Tsuboi N, et al. Human neutrophil FcyRIIA regulation by $\mathrm{C} 5 \mathrm{aR}$ promotes inflammatory arthritis in mice. Arthritis Rheum. 2011;63(2):467-478.

10. Galli SJ. Pathogenesis and management of anaphylaxis: current status and future challenges. J Allergy Clin Immunol. 2005;115(3):571-574.

11. Matasar MJ, Neugut AI. Epidemiology of anaphylaxis in the United States. Curr Allergy Asthma Rep. 2003;3(1):30-35

12. Neugut AI, Ghatak AT, Miller RL. Anaphylaxis in the United States: an investigation into its epidemiology. Arch Intern Med. 2001;161(1):15-21.

13. Cohen SG, Zelaya-Quesada M. Portier, Richet, and the discovery of anaphylaxis: a centennial. J Allergy Clin Immunol. 2002;110(2):331-336.

14. Portier P, Richet C. De l'actíon anaphylactíque de certaín veníns. CR Soc Biol (Paris). 1902;54:170-178.

15. Miyajima I, Dombrowicz D, Martin TR, Ravetch JV, Kinet JP, Galli SJ. Systemic anaphylaxis in the mouse can be mediated largely through IgG1 and Fc $\gamma$ RIII. Assessment of the cardiopulmonary changes, mast cell degranulation, and death associated with active or IgE- or IgG1-dependent passive anaphylaxis. J Clin Invest. 1997;99(5):901-914.

16. Tsujimura $Y$, et al. Basophils play a pivotal role in immunoglobulin-G-mediated but not immunoglobulin-E-mediated systemic anaphylaxis. Immunity.
2008;28(4):581-589.

17. Choi IH, et al. Immunoglobulin E-dependent active fatal anaphylaxis in mast cell-deficient mice. J Exp Med. 1998;188(9):1587-1592.

18. Oettgen HC, Martin TR, Wynshaw-Boris A, Deng C, Drazen JM, Leder P. Active anaphylaxis in IgEdeficient mice. Nature. 1994;370(6488):367-370.

19. Arias K, et al. Concurrent blockade of platelet-activating factor and histamine prevents life-threatening peanut-induced anaphylactic reactions. J Allergy Clin Immunol. 2009;124(2):307-314.

20. Nimmerjahn F, et al. Fc $\gamma$ RIV deletion reveals its central role for IgG2a and IgG2b activity in vivo. Proc Natl Acad Sci U S A. 2010;107(45):19396-19401.

\title{
Good COP1 or bad COP1? In vivo veritas
}

\section{Wenyi Wei ${ }^{1}$ and William G. Kaelin Jr. ${ }^{2,3}$}

${ }^{1}$ Department of Pathology, Beth Israel Deaconess Medical Center, Harvard Medical School, Boston, Massachusetts, USA. ${ }^{2}$ Department of Medical Oncology, Dana-Farber Cancer Institute, Harvard Medical School, Boston, Massachusetts, USA. ${ }^{3 H}$ oward Hughes Medical Institute, Chevy Chase, Maryland, USA.

\begin{abstract}
The evolutionarily conserved protein COP1 has been shown to operate as an E3 ubiquitin ligase complex, and a number of putative substrates have been identified, including the c-JUN oncoprotein and p53 tumor suppressor protein. New work by Migliorini and colleagues described in the current issue of JCI demonstrates that COP1 acts as a tumor suppressor in vivo and does so, at least in part, by promoting the destruction of $\mathrm{c}-J U N$. These findings challenge the view that COP1 regulates p53 stability and call into question the wisdom of developing COP1 inhibitors as potential anticancer agents.
\end{abstract}

Ubiquitin ligase complexes that target proteins for proteasomal degradation in a regulated manner play important roles in maintaining cellular homeostasis (1). Accordingly, deregulation of ubiquitin ligases and their protein targets has been implicated in a variety of diseases including cancer. For example, overexpression of MDM2, which ubiquitinates the p53 tumor suppressor protein, has been linked to certain cancers, as has loss of AXIN, which is a component of a ubiquitin ligase that targets the $\beta$-catenin oncoprotein for destruction. In this issue of JCI, Migliorini et al. provide evidence that loss of constitutive photomorphogenesis protein 1 (Cop1), a component of another ubiquitin ligase complex, leads to deregulation of the c-Jun oncoprotein and tumor growth in vivo.

COP1, also called RFW2 (RING finger and WD repeat domain 2), was initially identified in Arabidopsis, where it plays a critical role in growth and development in response to light (photomorphogenesis) (2). Under dark conditions, COP1 represses

Conflict of interest: The authors have declared that no conflict of interest exists.

Citation for this article: J Clin Invest. doi:10.1172/ JCI57080. photomorphogenesis by serving as an E3 ubiquitin ligase that targets proteins such as the photomorphogenesis-promoting transcription factor HY5 for destruction (3). In response to light, COP1 becomes inactive, both by virtue of translocation out of the nucleus and through inhibitory protein-protein interactions, thereby allowing photomorphogenesis to proceed.

\section{COP1 is an ubiquitin ligase}

Although mammalian cells do not undergo photomorphogenesis, they somewhat surprisingly also contain an Arabidopsis COP1 homolog. Mammalian COP1, like the Arabidopsis protein, consists of an $\mathrm{N}$-terminal RING finger domain, an internal coiledcoil domain, and C-terminal WD40 repeats. Moreover, mammalian COP1 possesses E3 ubiquitin ligase activity (4). In addition to possessing intrinsic E3 activity, COP1 participates, in both Arabidopsis and mammalian cells, in a higher-order E3 ubiquitin ligase complex that contains DET1 (deetiolated 1), DDB1 (DNA damage-binding protein 1), CUL4 (cullin 4), and RBX1 (ring-box 1) (4). The growing list of COP1 substrates identified so far includes p53 (5), c-JUN $(6,7)$, ACC1 (acetyl-coenzyme A carboxylase alpha) (8), MTA1 (metastasis- associated protein 1) (9), FOXO1 (forkhead box protein O1) (10), TORC2 (transducer of regulated CREB activity 2) (11), and PEA3 (polyomavirus enhancer activator-3) (12). The evidence for these being COP1 targets rests entirely on biochemical and cell culture experiments, raising questions as to which are actually regulated by COP1 in vivo. Moreover, inclusion of the p53 tumor suppressor protein and the c-JUN oncoprotein as potential COP1 targets made it difficult to predict whether COP1 would serve primarily as an oncoprotein or as a tumor suppressor (Figure 1), despite recent calls to develop COP1 inhibitors for use as cancer therapeutics.

\section{Cop1 regulates c-Jun and functions as a tumor suppressor in vivo}

To more fully understand the physiological roles of COP1, Migliorini and colleagues utilized a genetic approach to generate an allelic series of Cop1-mutant mice (13). The use of hypomorphic (partial-loss-of-function) alleles is a potentially powerful way to circumvent lethality associated with null alleles and also allows one to interrogate the doseresponse relationships linking gene activity to functional outputs. The methodology employed by Migliorini and coworkers can be used with genes that have been targeted with pGT0lxf or pGT0lxr vectors, available through the International Gene Trap Consortium (http://www.genetrap.org).

The authors found that c-Jun abundance, but not p53 abundance or activity, was elevated in Cop1-hypomorphic mice that expressed only $10 \%$ of Cop 1 compared with the wild-type counterparts (13). Increased 